\title{
Hamartomatous Nodule, Sertoli Cell Adenoma in Complete Androgen Insensitivity Syndrome with Wolffian/Müllerian Duct Remnants: An Unusual Case Report
}

\author{
Prashant Vijay Kumavat ${ }^{1 *}$, Chetan S Chaudhari ${ }^{1}$, Anita Padmanabhan ${ }^{1}$, Nitin M Gadgil ${ }^{1}$, \\ Sangita S Margam ${ }^{1}$, Ganesh R Kshirsagar ${ }^{1}$ and Unnati D Rathod ${ }^{2}$ \\ ${ }^{1}$ Dept. Of Pathology, LTMMC and LTMGH Sion, Mumbai, India \\ ${ }^{2}$ Dept. Of Microbiology, Sir JJ Hospital, Byculla, Mumbai, India
}

\begin{abstract}
Complete Androgen insensitivity syndrome is a disorder of hormone resistance characterized by a female phenotype with an XY karyotype and testes producing age-appropriate normal or higher concentrations of androgens. We present a case of 26 year old, unmarried phenotypically female, with left inguinal swelling and amenorrhea. MRI finding revealed bilateral inguinal masses, uterus cervix was not visualized and hypoplatic vagina was noted. Karyotyping revealed her genotype as $46 \mathrm{XY}$. Hormonal investigation showed testosterone, estradiol and LH were increased and FSH was within normal limits. Patient underwent laparoscopic bilateral gonadectomy with left open hernia repair. Histopathology examination revealed hamartomatous nodule, sertoli cell adenoma, leydig cell hyperplasia, which are more pronounced as age advances as result of absent activity of androgen. Fallopian tube, underdeveloped vas deference, Wolffian/Müllerian cysts lined by cuboidal epithelium was also noted which may be reminiscent of Wolffian/ Müllerian structure. Immunostaining for PLAP and CD 117 were negative. The clinical, MRI, laboratory and histopathology findings confirmed diagnosis of complete androgen insensitivity syndrome.
\end{abstract}

Keywords: Complete Androgen Insensitivity Syndrome, Gonads, Hamartoma, Müllerian, Wolffian.

\section{Introduction}

Complete androgen insensitivity syndrome (CAIS) is a female phenotype with a male karyotype (46, XY) which results from inactivating mutation in the androgen receptor (AR) gene. ${ }^{[1]}$ The targeted response to testosterone or dihydrotestosterone is annulled due to mutation. As a result, male external genitalia differentiation and Wolffian duct development do not occur correctly. Sertoli cells of normally developed gonads produces anti-Mullerian hormone which regresses Mullerian duct. Residual Müllerian structures exist approximately in one third of patients. ${ }^{[2]}$ Development of the gonads is normal, and serum androgen level is comparable with that of a normal male. Most of the patients with complete androgen insensitivity typically presents either at puberty with primary amenorrhea or before puberty with masses in the inguinal canal that are subsequently identified as testes. Breast development occurs because of the aromatization of the excess testosterone into oestrogen and pubic hair tends to be sparse or absent. External genitalia are that of female with short vagina and absent uterus, cervix, or fallopian tubes. After development of secondary sexual features, testes either in the inguinal canals or in the pelvis should be removed, because gonadal tumours are known to develop in $5 \%$ of cases. ${ }^{[3]}$ We report a case of complete androgen insensitivity syndrome in twenty six year old unmarried female but found to have a 46, XY genotype, with hamartomatous nodule, sertoli cell adenoma and residual Müllerian/Wolffian tissue.

\section{Case report}

A young unmarried, 26 year old female, presented to surgery OPD with left inguinal swelling increasing on straining and coughing, since 4 years. She was amenorrheic but never consulted for this complaint to physician. She had past history of right inguinal hernia operated four years back. MRI finding revealed bilateral inguinal masses, on right side, $2.3 \times 1.1 \mathrm{~cm}$ mass anterior to external iliac vessel and similarly on left side, and $2 \times 1 \mathrm{~cm}$ anterior to external iliac vessel (Figure 1 A, B).Uterus cervix was not visualized and a small tubular structure was noted between urethral and anal opening, suggestive of hypoplatic vagina. She has not attained menarche but has attained thelarche (breast development), 12 years back. On local examination atrophic vagina and clitoris was present and axillary, pubic hair growth was absent. Karyotyping was advised and revealed her genotype as $46 \mathrm{XY}$. Hormonal investigation showed, testosterone level of $251.4 \mathrm{ng} / \mathrm{dl}$ (increased), Follicle stimulating hormone (FSH) level of $9.88 \mathrm{mIU} /$ $\mathrm{ml}$ (normal), Luteinizing hormone (LH) of $82.84 \mathrm{mIU} /$ $\mathrm{ml}$ (increased), estradiol of $413 \mathrm{pg} / \mathrm{ml}$ (increased) and 
progesterone of $0.19 \mathrm{ng} / \mathrm{ml}$ (decreased). Intraoparative findings revealed absence of ovaries and uterus and bilateral small atrophied gonads were present. Widened left sided deep inguinal ring, with left sided gonads partially herniating was observed.Patient underwent laparoscopic bilateral gonadectomy with left open hernia repair.

Grossly, we received left inguinal mass measuring $6 \times 3 \times 2$ $\mathrm{cm}$, with cut section showing grey white nodule measuring $1.2 \times 1.0 \mathrm{~cm}$ and right sided inguinal mass measuring $5 \times 3 \times 2$ $\mathrm{cm}$ with cut surface showing grey white in appearance. (Figure 1C). Histopathology examination of both masses showed hamartomatous nodule of sertoli and leydig cells, which is well circumscribed and composed of sertoli cells tubules and leydig cell in between tubule and leydig cell hyperplasia (Figure 2 A). At many places fibrotic stroma with atrophic seminiferous tubule were noted (Inset Figure 2 A). Areas of ovarian like stroma were noted (Inset Figure 2B). Sertoli cell adenoma showing Somniferous tubules having little fruitless lumens and typically containing just sertoli cells were also noted (Figure 3 A, B). There was no evidence of spermatogenesis in any of the structures and no apparent malignant changes. Fallopian tube, underdeveloped vas deference, and Wolffian/ Müllerian duct cysts lined by cuboidal epithelium were also noted (Figure $3 \mathrm{C}, \mathrm{D})$. IHC revealed negative immunostaining for PLAP and CD 117(Figure $3 \mathrm{E}, \mathrm{F}$ ). The clinical, MRI, laboratory and histopathology findings confirmed the diagnosis of complete androgen insensitivity syndrome

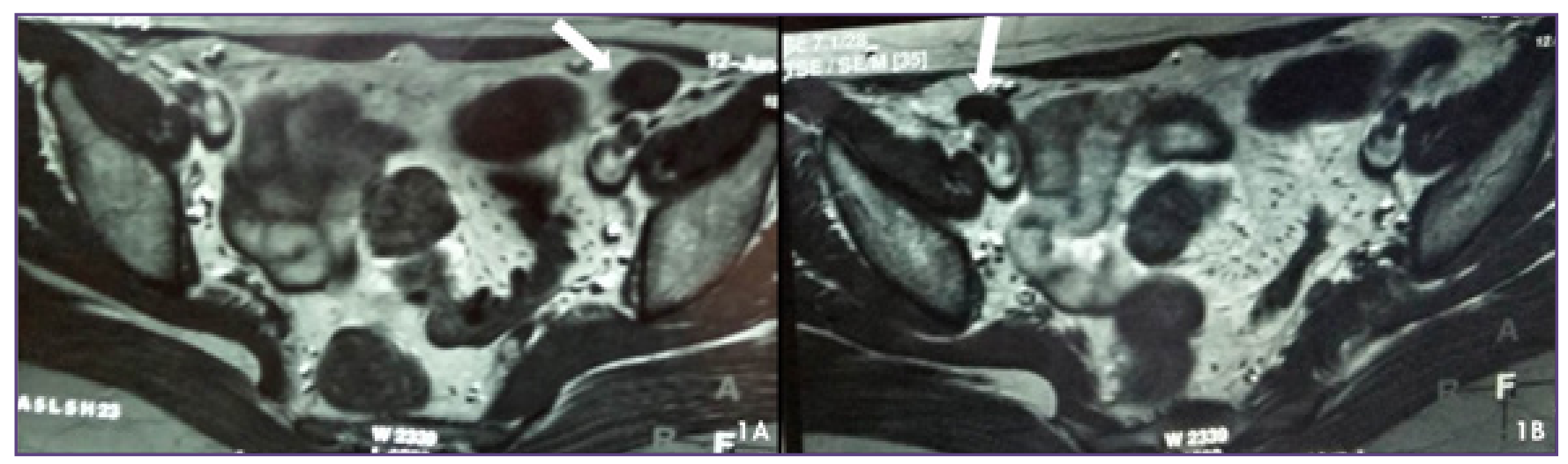

Fig. 1: 1A MRI Scan $-2.3 \times 1.1 \mathrm{~cm}$ inguinal mass anterior to external iliac vessel on right side. $1 \mathrm{~B} \mathrm{MRI}-2 \times 1 \mathrm{~cm}$ on left side anterior to external iliac vessel.

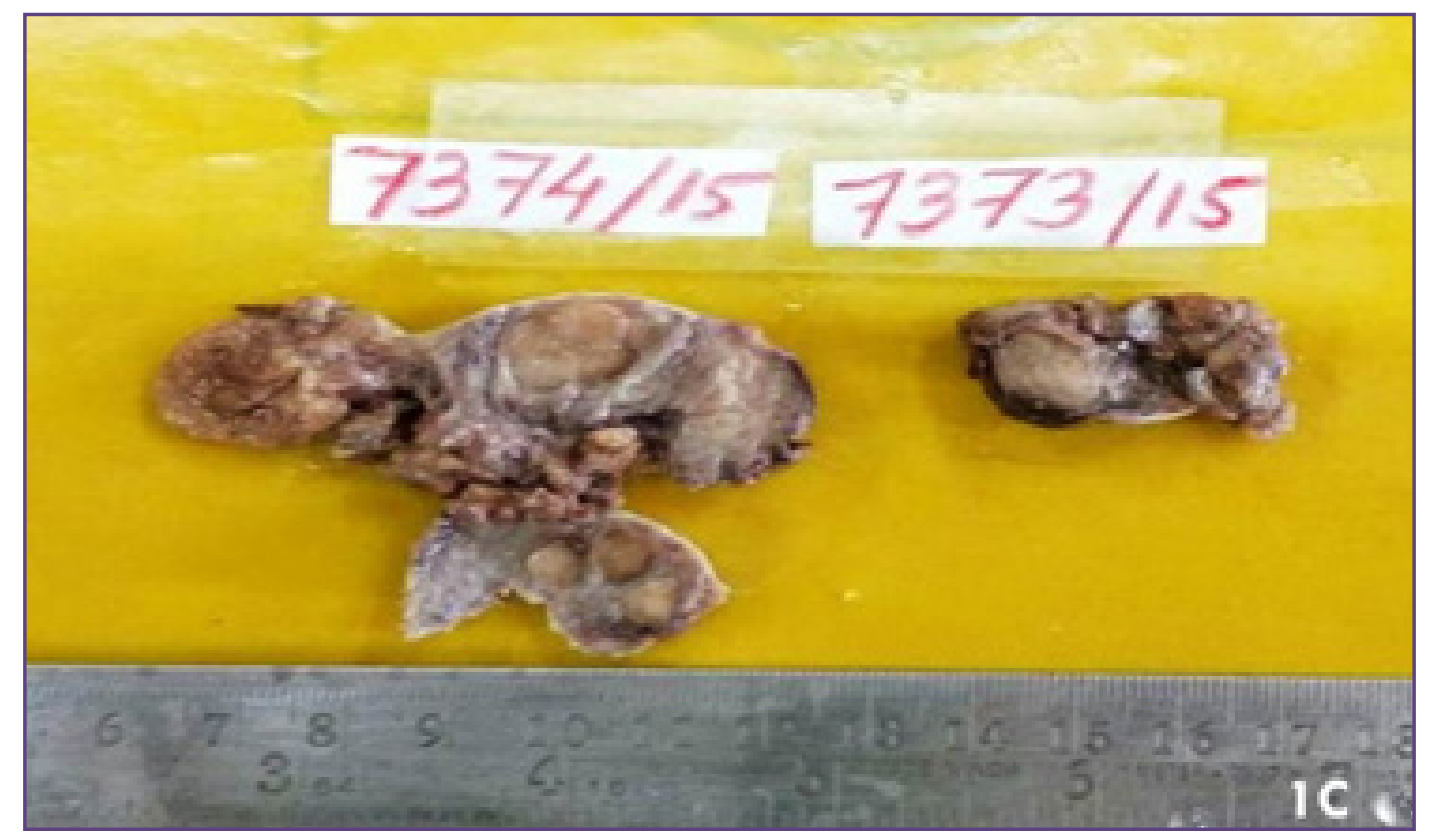

Fig. 1C: Gross- Left mass measures $6 \times 3 \times 2 \mathrm{~cm}$, cut sections showed grey white nodule measuring $1.2 \times 1 \mathrm{~cm}$. Right sided mass measures $5 \times 3 \times 2 \mathrm{~cm}$ and cut section showed grey white. 


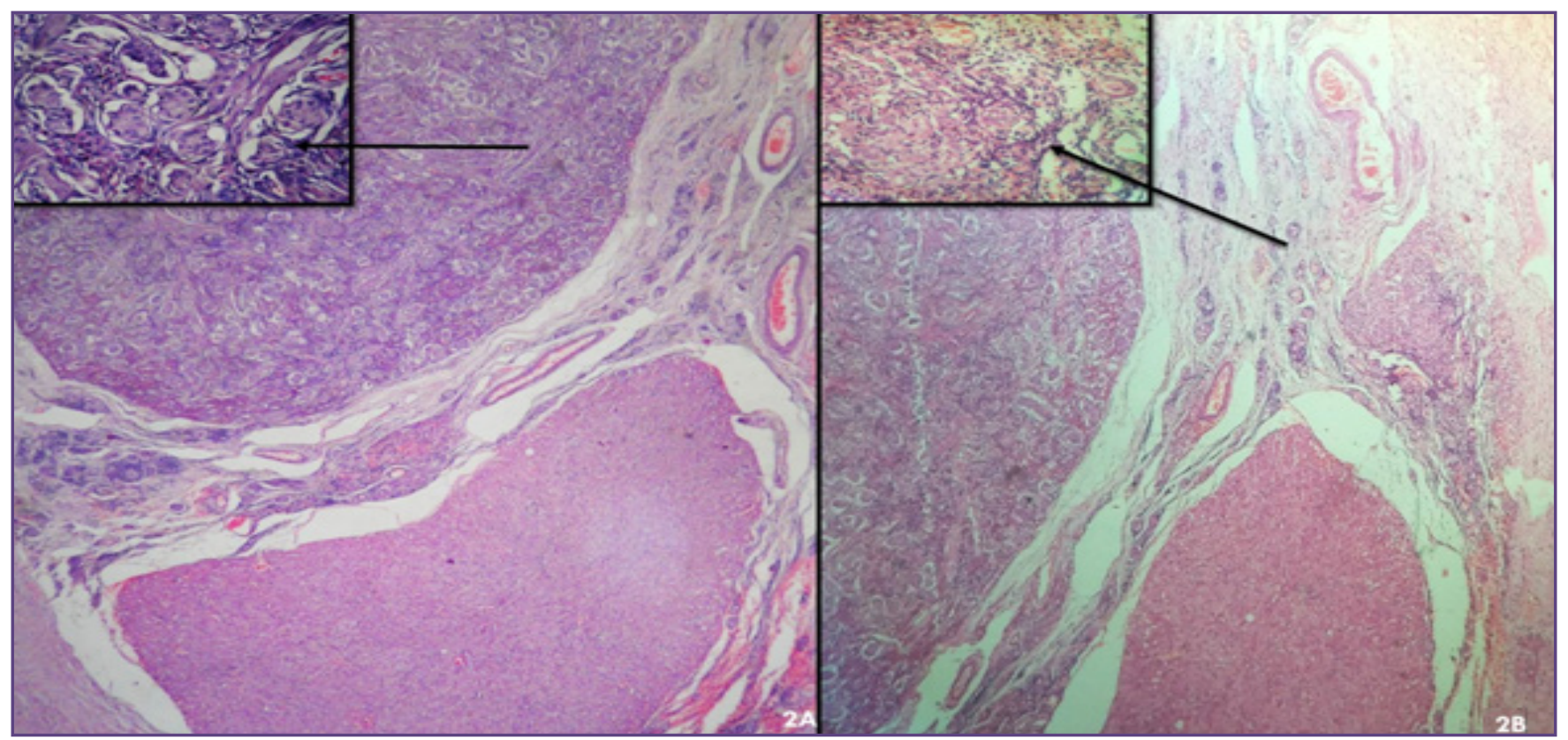

Fig. 2: 2A- Well circumscribed Hamartomatous nodule of sertoli and leydig cells and leydig cell hyperplasia (H and E 100x) Inset - At many places fibrotic stroma with atrophic seminiferous tubule were noted. 2B- Inset- Areas of ovarian like stroma was noted.

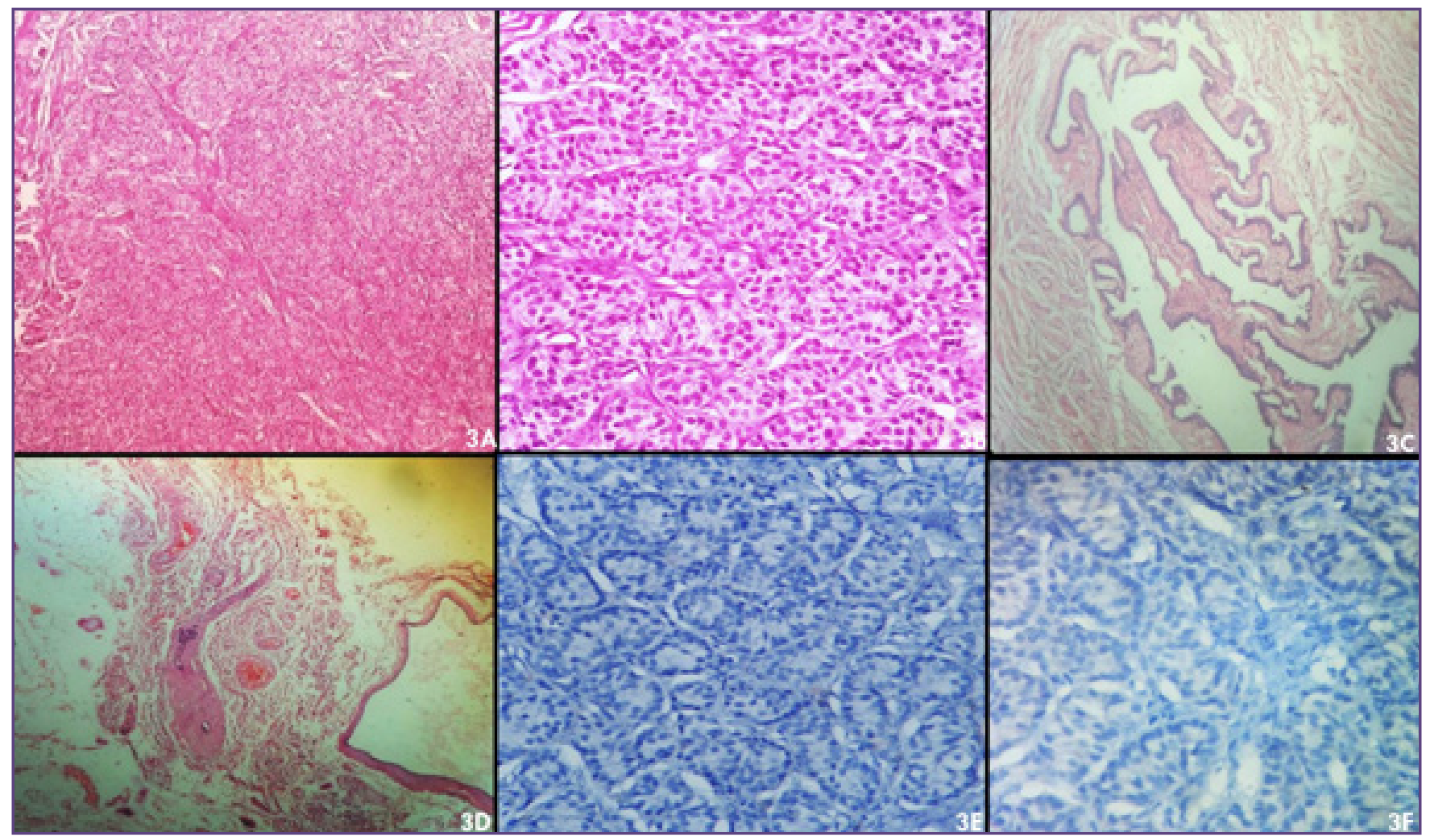

Fig. 3: 3A- Sertoli cell adenoma - Seminiferous tubules have little fruitless lumens and just sertoli cells (H and E 100x). 3B- High power of 3A (H and E 400x). 3C- Fallopian tube like structure. 3D - underdeveloped vas deference and Wolffian/ Müllerian duct cysts lined by cuboidal epithelium. 3E- CD 117 negative. 3F- PLAP negative. 


\section{Discussion}

Androgen insensitivity syndrome (AIS) is an X-linked, male pseudo hermaphroditism also known as testicular feminization syndrome, in which clinically phenotypically patient is female but shows $46 \mathrm{XY}$ genotype. The frequency of this syndrome was estimated about $0.05 \%$ ( 70/140000) ${ }^{[4]}$ In post pubertal patients, the most frequent cause for consultation is primary amenorrhea; however, in prepubertal patients, AIS is often diagnosed during the investigation of inguinal hernia. ${ }^{[5]}$ There are 3 types of ASI: complete, mild and incomplete. The estimated prevalence of CAIS is about 1:20,000-64,000 male births. ${ }^{[6]}$ In most of the cases CAIS, phenotypically female present with normal female external genitalia like a short blind ending vagina but there will be an absence of Wolffian duct derived structures like vas deferens and seminal vesicles epididymis and prostate. Although pubic and axillary hair are sparse or absent but breast development is recognised at puberty. ${ }^{[6]}$

The histologic pattern of testes removed from adult patients with AIS is similar to that of many cryptorchidic, infantile or immature testes. ${ }^{[7]}$ Although some differences regarding testicular histology between the complete and incomplete forms of AIS were reported in the first studies ${ }^{[6]}$ later studies of a wide series of patients concluded that there are no histologic differences in the testicular pattern between the 2 forms.

Rutgers and Scully reported two histopathological testicular patterns in AIS, namely, hamartomas (hamartomatous nodules) and sertoli cell adenomas which are seen in our case also. ${ }^{[7]}$ Hamartomas consisted of solid tubules filled with immature sertoli cells, prominent leydig cells, and rare fascicles of smooth muscle. Multiple hamartomas occurred in $63 \%$ of AIS cases (bilateral in $40 \%$ of cases). Sertoli cell adenomas consisted of small tubules that were filled by immature sertoli cells and scant leydig cells and occurred in $23 \%$ of cases. In the present case, hamartomatous nodule was seen with sertoli cell adenoma and leydig cell hyperplasia. We propose that the development of hamartomas in AIS is not neoplastic proliferation of sertoli cells but it results from malformative process of the congenitally hypoplastic seminiferous tubules.

Regression of the Müllerian structures occurs (the fallopian tubes, uterus, and upper portion of the vagina) because of AMH (Anti- Müllerian hormone) secreted by sertoli cells of the testis. The incomplete regression of Müllerian remnants can be due to 1) Deficiency of secretion of $A M H, 2) A M H$ although secreted enough but it's not functional, 3) Lack of response by the Müllerian tissue to AMH because of the high oestrogen levels caused by the conversion of testosterone to oestrogen in the androgen insensitive foetus, 4) Early testicular descent that removes the Müllerian structures out of the effective range of the AMH. ${ }^{[6,8,9]}$ There is a possibility of common link between androgen insensitivity syndrome and defective action of $\mathrm{AMH}$, this can be suggested as there are persistence of the Müllerian remnants in patients diagnosed with complete androgen insensitivity syndrome. ${ }^{[10]}$ In one of the studies from Netherlands (one of the largest databases compiled) of the families examined revealed 3 of 7 CAIS families had female siblings with differing Müllerian remnants. ${ }^{[11]}$ One third of the case showed fallopian tube which is also noted in our case. ${ }^{[7]}$ One more interesting fact seen in our case is presence of underdeveloped vas deference that may be reminiscent of Wolffian duct. Wolffian/Müllerian duct cysts lined by cuboidal epithelium was also noted which may be reminiscent of that structure. Ovarian like stroma was present in our cases. ${ }^{[12]}$ So this is very rare case showing finding of CAIS with discordant Wolffian/ Müllerian remnants, offering another example and more studies should be initiated and evaluated to study the cause leading to the residual tissue.

Some histopathological changes seen in our case that is Hamartomatous nodule, sertoli cell adenoma and leydig cell hyperplasia develop during puberty as a consequence of the almost or entirely absent activity of androgens in complete androgen insensitivity syndrome. ${ }^{[12]}$ Decrease or absent germ cells, tubular atrophy, stromal atrophy occur early in childhood which may be due to abnormal location (inguinal) of the gonads. ${ }^{[12]}$

Intratubular germ cell neoplasia, can be diagnosed only if at least one cross section of seminiferous tubule contains a homogenous population of atypical germ cell with angulated nuclei. ${ }^{[12]}$ This particular feature was absent in our case, which may be due to absolute loss of abnormal germ cells in adulthood when gonads would have been retained and a failure of progression of the pre-invasive lesions into an invasive cancer. In CAIS both these above mentioned mechanism may be due to lack of androgen action because of which there is risk of tumour development in patients with partial AIS as compared to CAIS (15\% versus $0.8 \%$ in complete androgen insensitivity syndrome according to Cools et al). ${ }^{[13]}$

Due to alteration in androgen receptor gene which causes end organ resistance to the testosterone, leading to testosterone and LH levels are usually elevated in AIS. ${ }^{[6]}$ In AIS excess of testosterone is converted to oestrogen in periphery which causes increase levels of estradiol, while FSH levels are normal. This suggests that regulation of FSH secretion is maintained by the combine action of estradiol and gonadal hormone like Inhibin. ${ }^{[14]}$ 
In conclusion Hamartomatous nodule, sertoli cell adenoma, leydig cell hyperplasia is more pronounced as age advances as result of absent activity of androgen. This is a very rare case showing finding of CAIS with discordant Wolffian/Müllerian remnants, and more studies should be initiated and evaluated to study the cause leading to the residual tissue.

Lack of androgen theory would correlate with significantly higher risk for tumor development in patient with partial androgen insensitivity syndrome as compared to complete androgen insensitivity syndrome.

\section{References}

1. Hughes I, Davies J, Bunch T, Pasterski V, Mastroyannopoulou $\mathrm{K}$, MacDougall J. Androgen insensitivity syndrome. The Lancet. 2012;380(9851):1419-1428.

2. Nichols J, Bieber E, Gell J. Case of sisters with complete androgen insensitivity syndrome and discordant Müllerian remnants. Fertility and Sterility. 2009;91(3):932.e15-932.e18.

3. Chen C, Chen S, Wang T, Wang W, Hwu Y. A frame shift mutation in the DNA-binding domain of the androgen receptor gene associated with complete androgen insensitivity, persistent müllerian structures, and germ cell tumours in dysgenetic gonads. Fertility and Sterility. 1999;72(1):170-173.

4. D Farhud D, Zarif Yeganeh M, Sadighi H, Zandvakili S. Testicular Feminization or Androgen Insensitivity Syndrome (AIS) in Iran: a Retrospective Analysis of 30-Year Data. Iranian Journal of Public Health. 2016;45(1):1-5.

5. Bangsbøll S, Qvist I, Lebech P, Lewinsky M. Testicular feminization syndrome and associated gonadal tumours in Denmark. Acta Obstetricia et Gynecologica Scandinavica. 1992;71(1):63-66.

6. Quigley C, Bellis A, Marschke K, El-Awady M, Wilson E, French F. Androgen Receptor Defects: Historical,
Clinical, and Molecular Perspectives. Endocrine Reviews. 1995;16(3):271-321.

7. Rutgers J, Scully R. The Androgen Insensitivity Syndrome (Testicular Feminization). International Journal of Gynaecological Pathology. 1991;10(2):126-144.

8. Heller D, Ranzini A, Futterweit W, Dottino P, Deligdisch L. Müllerian remnants in

9. complete androgen insensitivity syndrome. International journal of fertility. 1992;37(5):283.

10. Ulloa-Aguirre A, Mendez J, Chavez B, Carranza-Lira S, Angeles A, Perez-Palacios G. Incomplete regression of Müllerian ducts in the androgen insensitivity syndrome. Fertility and Sterility. 1990;53(6):1024-1028.

11. Damiani D, Mascolli M, Almeida M, Jaubert F, Fellous M, Dichtchekenian V et al. Persistence of Mullerian Remnants in Complete Androgen Insensitivity Syndrome. Journal of Paediatric Endocrinology and Metabolism. 2002;15(9):1553-1556.

12. Boehmer A, Brüggenwirth $\mathrm{H}$, Van Assendelft C, Otten B, Verleun-Mooijman M,

13. Niermeijer $M$ et al. GenotypeversusPhenotype in Families with Androgen Insensitivity Syndrome. The Journal of Clinical Endocrinology \& Metabolism. 2001;86(9):4151-4160.

14. Kaprova-Pleskacova J, Stoop H, Brüggenwirth $H$, et al. Complete androgen insensitivity syndrome: factors influencing gonadal histology including germ cell pathology. Modern Pathology. 2014;27(5):721-30.

15. Cools M, Drop S, Wolffenbuttel K, Oosterhuis J, Looijenga L. Germ Cell Tumours in the Intersex Gonad: Old Paths, New Directions, Moving Frontiers. Endocrine Reviews. 2006;27(5):468-484.

16. Schmitt S, Knorr D, Schwarz H, Kuhnle U. Gonadotropin regulation during puberty in complete androgen insensitivity syndrome with testicles in situ. Hormone Research. 1994;42(6):253-256.

*Corresponding author:

Dr Prashant Vijay Kumavat, Room no 1303, building no 27, hawre city, kasarvadvali, Thane (w) 400615

Email: drkumavat_83@rediffmail.com

Financial or other Competing Interests: None. 